JGG 2021;69:215-217

doi: $10.36150 / 2499-6564-N 341$

\title{
Emphysematous cystitis in an elderly male diabetic patient: a case report. A complicated urinary tract infection
}

Annalisa Paglia, Anna Grazia Angeletti, Vera Conte, Gaetano Serviddio, Antonino Davide Romano

Department of Medical and Surgical Sciences, Division of Geriatrics and Gerontology, University of Foggia, Foggia, Italy

Emphysematous Cystitis $(E C)$ is a rare form of complicated urinary tract infection, characterized by the presence of gas in the bladder and in its wall. Diabetes Mellitus (DM) is the major risk factor. Clinical manifestations in EC can range from asymptomatic form to severe sepsis and fatal events. Abdominal imaging is requested to diagnose EC and evaluate its severity. Early diagnosis and appropriate management improve the outcome. We report a case of incidental EC diagnosis in an 82-yearold diabetic male who was investigated for severe anemia.

Key words: emphysematous cystitis, urinary tract infection, elderly, diabetes

Received: January 07, 2021

Accepted: March 09, 2021

\section{Correspondence}

\section{Gaetano Serviddio}

Department of Medical and Surgical Sciences,

Division of Geriatrics and Gerontology, University of Foggia, viale Luigi Pinto 1, 71122 Foggia,

Italy. E-mail: gaetano.serviddio@unifg.it

How to cite this article: Paglia A, Angeletti AG, Conte V, et al. Emphysematous cystitis in an elderly male diabetic patient: a case report. A complicated urinary tract infection. Journal of Gerontology and Geriatrics 2021;69:215-217. https://doi. org/10.36150/2499-6564-N341

(C) Copyright by Società Italiana

di Gerontologia e Geriatria (SIGG)

\section{(c) (1) () $(9$}

\section{OPEN ACCESS}

This is an open access article distributed in accordance with the CC-BY-NC-ND (Creative Commons Attribution-NonCommercial-NoDerivatives 4.0 International) license. The article can be used by giving appropriate credit and mentioning the license, but only for non-commercial purposes and only in the original version. For further information: https://creativecommons.org/licenses/by-nc-nd/4.0/deed.en

\section{INTRODUCTION}

Emphysematous Cystitis (EC) is a rare form of complicated urinary tract infection, characterized by the presence of gas in the bladder and in its wall, in the absence of both bladder fistula and iatrogenic pneumaturia ${ }^{1,2}$. Gas-forming microbes are involved. EC is most frequently caused by Escherichia coli (60\% of cases) and Klebsiella pneumoniae (10-20\%) ${ }^{1,3}$. The major risk factor is Diabetes Mellitus (DM), and it is estimated that $60-70 \%$ of EC cases occur in diabetic patients. Other risk conditions of EC are bladder dysfunctions, immune system impairment, urinary catheterization. Women are more affected than men, and the incidence of EC increases with age ${ }^{1,4,5}$. Clinical manifestations in EC can range from asymptomatic form to severe sepsis and fatal events. Mortality attributed to this condition is about $20 \%{ }^{2,4}$. In asymptomatic patients (approximately $7 \%$ of cases) diagnosis is usually incidental. Affected patients can present abdominal pain. Urinary tract symptoms as dysuria, urinary frequency and urinary urgency charactherise almost $50 \%$ of cases. Hematuria is present in $60 \%$ of EC. Even if it is not frequently reported, pneumaturia can be observed in $70 \%$ of catheterized patients. Fever usually detects complications as emphysematous pyelonephritis (EP), with higher rates of mortality. In 50\% of cases blood culture is positive for bacteremia ${ }^{1,6,7}$. Abdominal imaging is requested to diagnose $\mathrm{EC}$ and evaluate its severity. Abdominal $\mathrm{X}$-ray (AXR) and ultrasound (AUS) are the most common first-line methods, while Abdominal Computed tomography (ACT) is the most sensitive exam regarding diagnosis and severity assessment ${ }^{1,8}$. Urine culture gives information about the responsible bacteria, guiding antibiotic treatment. 
Early diagnosis and antibiotic therapy of EC improve prognosis. Surgery is reserved to severe cases that are resistant to pharmacological treatment or in the event of complications such as bladder perforation 1,9,10.

\section{CASE PRESENTATION}

An 82-year-old man was brought to hospital for severe anaemia showed up in routine tests $(\mathrm{Hb} 7.5 \mathrm{~g} /$ dl, 13-17), that required the transfusion of two units of WBC-depleted RBCs in Emergency Department. After clinical stabilisation, he was admitted to post-acute care Geriatric Unit. The patient was affected by Type II DM (T2DM) in treatment with an oral anti-diabetic agent in association to long-acting insulin, chronic ischemic heart disease, chronic hepatitis $\mathrm{C}$ and chronic infiltrative interstitial lung disease. Three months earlier he was hospitalized for an episode of acute coronary syndrome, with a prolonged urinary catheterization. The physical examination was unremarkable: he had normal vital signs, with normal temperature; abdomen was soft, non-tender. Laboratory tests revealed microcytic hypochromic anemia with low levels of serum iron (16 ng/ ml, 60-160); neutrophilic leukocytosis (WBC 11180/uL, $67.3 \%$ neutrophils); C-reactive protein (CRP) $1.6 \mathrm{mg} / \mathrm{L}$ (0-5); erythrocyte sedimentation rate $32 \mathrm{~mm} / \mathrm{h}$ (1-18); blood glucose 102 mg/dl (50-110). Glycated hemoglobin value (HbA1c) was $46 \mathrm{mmol} / \mathrm{mol}$ (20-38). Kidney function test was normal. Urinalysis showed hemoglobinuria (> $200 \mathrm{mg} / \mathrm{dl}$ ), proteinuria $(100 \mathrm{mg} / \mathrm{dl}$ ), presence of nitrites and leucocytic esterase. Urine sediment presented non-specific findings, with high concentration of bacteria (1712/ul), white blood cells and red blood cells. Patient was asymptomatic for urinary tract disorders. Six days after admission, he reported an episode of macrohematuria. Urine culture revealed a positivity to infection by Klebsiella pneumoniae with multidrug resistance at the antibiogram test, producing extended spectrum beta-lactamase. Serum levels of CPR raised (117.7 mg/L) and procalcitonin was $0.7 \mathrm{ng} / \mathrm{ml}(<0.5)$, with a negative blood culture result. Patient underwent to CT of thorax and abdomen (Fig. 1) to investigate anaemia. The exam showed parietal pneumatosis of the bladder, and a wide fluid level made of air and urine occupying the upper third of bladder lumen. Moreover, patient started reporting symptoms of pneumaturia and urinary frequency, so a five days antibiotic therapy with intravenous $1 \mathrm{~g}$ of meropenem three times a day was commenced. Rectoscopy showed haemorrhoidal disease and no recto-bladder fistulae. Cystography (Fig. 2) revealed a condition of stress bladder, with no filling defect or contrast agent leakage. A second urine culture was made 48 hours after the end of antibiotic therapy,

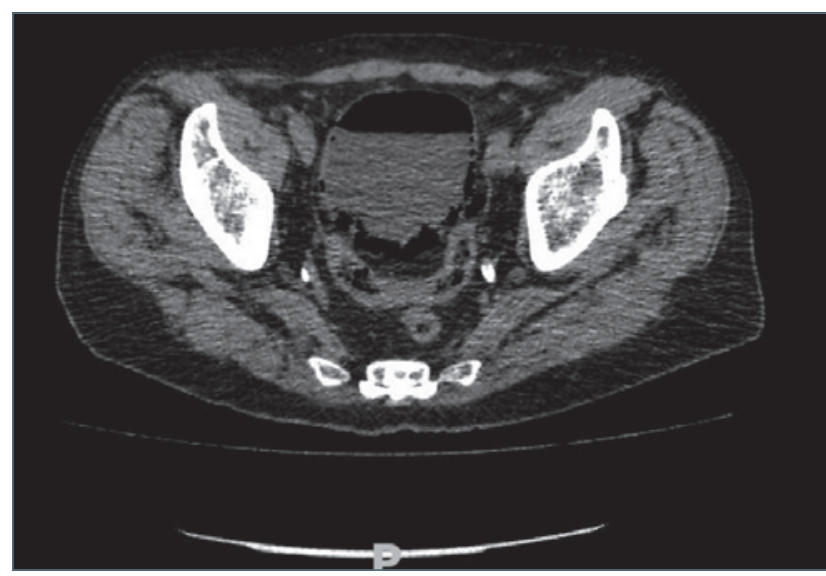

Figure 1. Axial abdomen CT scan, showing parietal pneumatosis of the bladder.

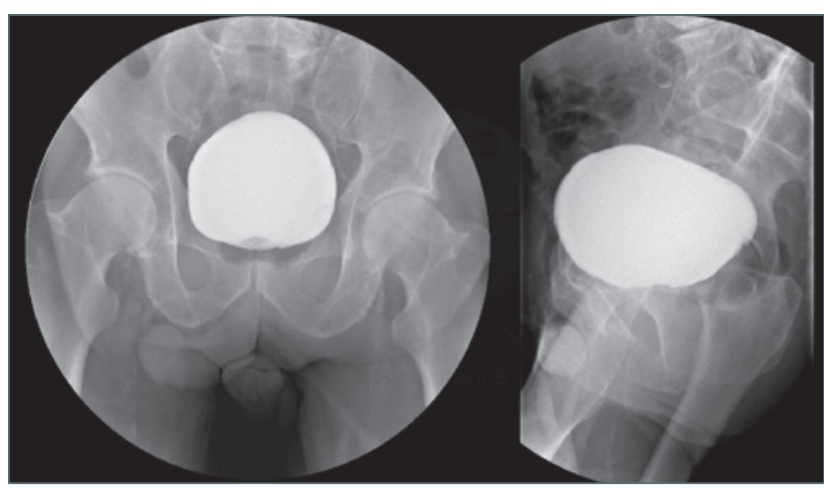

Figure 2. Cystography showing no filling defect or contrast agent leakage.

which resulted negative to infections. The patient was then discharged, with a scheduled clinical and diagnostic follow up for his anaemia.

\section{CONCLUSIONS}

EC is a relatively rare complication of urinary tract infection, mostly occurring in patients affected by DM. EC clinical presentation is variable and with high risk of complications. Diabetic patients with urinary tract infection presenting abdominal pain and hematuria should be studied with further imaging. Our case report prompts the usefulness of a first-line diagnostic protocol even in asymptomatic diabetic patient with laboratory sign of infection, since early diagnosis and appropriate treatment lead to a better clinical outcome. Elderly diabetic patients need particular attention, because age works as an additional risk factor for EC as well as frailty and presence of comorbidities. 


\section{Ethical consideration}

None.

\section{Acknowledgement}

None.

\section{Funding}

None.

\section{Conflict of interest}

The Authors declare no conflict of interest.

\section{Author contributions}

All the authors contributed in the development of this manuscript.

\section{References}

1 Amano M, Shimizu T. Emphysematous cystitis: a review of the literature. Intern Med 2014;53:79-82. https://doi.org/ 10.2169/internalmedicine.53.1121

2 Abusnina W, Shehata M, Nassri S, et al. Emphysematous cystitis. Cleve Clin J Med 2019;86:10-11. https://doi.org/ 10.3949/ccjm.86a.18063

3 Mokabberi R, Ravakhah K. Emphysematous urinary tract infections: diagnosis, treatment and survival (case review series). Am J Med Sci 2007;333:111-116. https://doi. org/10.1097/00000441-200702000-00009
4 Thomas AA, Lane BR, Thomas AZ, et al. Emphysematous cystitis: a review of 135 cases. BJU Int 2007;100:17-20. https://doi.org/10.1111/j.1464-410X.2007.06930.x

5 Ho MP, Chou A-H, Wu Y-H, et al. Emphysematous cystitis in an elderly diabetic patient. Geriatr Gerontol Int 2017;7:1027-1028. https://doi.org/10.1111/ggi.12980

6 Schicho A, Stroszczynski C, Wiggermann P. emphysematous cystitis: mortality, risk factors, and pathogens of a rare disease. Clin Pract 2017;7:930. https://doi.org/10.4081/ cp.2017.930

7 Li S, Wang J, Hu J, et al. Emphysematous pyelonephritis and cystitis: a case report and literature review. J Int Med Res 2018;46:2954-2960. https://doi. org/10.1177/0300060518770341

8 Wang $Q$, Sun $M$, Ma C, et al. Emphysematous pyelonephritis and cystitis in a patient with uremia and anuria: a case report and literature review. Medicine (Baltimore) 2018;97:e11272. https://doi.org/10.1097/ MD.0000000000011272

9 Hudnall MT, Jordan BJ, Horowitz J, et al. A case of emphysematous cystitis and bladder rupture. Uro Case Rep 2019;24:100860. https://doi.org/10.1016/j. eucr.2019.100860

10 Rajaian S, Murugavaithianathan P, Krishnamurthy K, et al. Emphysematous cystitis: diagnosed only if suspected. BMJ Case Rep 2019;12:e230715. http://dx.doi. org/10.1136/bcr-2019-230715 